\title{
SKIN THRUSH IN EARLY INFANCY
}

\author{
BY \\ A. HOLZEL \\ From the Department of Child Health, University of Manchester, and the Duchess of York Hospital for Babies, \\ Manchester
}

(RECEIVED FOR PUblication JUNE 1, 1953)

Thrush is generally regarded as one of the rarer infections of the skin of the newborn and young infant, but its occurrence is probably more frequent than its recognition. The high susceptibility of the oral mucosa to Monilia albicans during the early weeks of life is well known and it would be surprising were it limited to this structure. Invasion of the alimentary tract and other internal organs has been reported by Ludlam and Henderson (1942) and by Lederer and Todd (1949). Pulmonary moniliasis has assumed greater significance since the advent of antibiotic therapy and has received considerable attention during the past few years.

Nikolowski (1953) recently pointed to the danger of generalized moniliasis in adult patients who, suffering from a localized mycosis, had for one reason or another to undergo treatment with large doses of an antibiotic. This danger also exists in infancy. Moniliasis of the skin in infants may appear sporadically or in small epidemic outbreaks in institutions such as maternity nurseries or babies hospitals. Mayer, Götz and Seitz (1951) reported an epidemic in $\mathbf{4 0}$ infants under the age of 5 months which presented itself under the picture of erythrodermia desquamativa (Leiner), intertrigo and seborrhoeic dermatitis, involving also the scalp. The disease started in each case in the anal region and spread from there upwards or downwards. Of 35 babies who were examined bacteriologically, 17 had Monilia albicans on the skin and in 30 it was found in the stools. The incubation period was estimated as lasting from 14 to 28 days.

Moniliasis of the skin in infants is commonly accepted as associated with thrush of the oral cavity and as arising in the perianal area whence it spreads to other parts of the body. The faeces are regarded as the usual source of infection.

The primary lesion often appears as a small, flat, brownish-red papule without any inflammatory reaction in the surrounding skin. Later desquamation occurs on the surface of the papule and at its periphery, so that the lesion is surrounded by a characteristically rolled-in epithelial border. An alternative early manifestation is a small vesicle, which ruptures after a short time, the epithelium again forming the typical border which surrounds an erythematous area denuded of its epithelial covering. When several of these foci merge larger areas of the skin may be involved and present a typical clinical picture. Difficulty, however, may arise if the infection is superimposed on other skin manifestations not primarily related to the Monilia albicans infection.

During the past year we had opportunity to observe 11 cases of skin thrush in young infants, four of which were noticed practically simultaneously in a minor outbreak in a unit for premature infants, one premature infant was admitted from a maternity unit with an extensive rash at the age of 4 weeks, and six others were seen at different times in a clinic for well babies. These cases differ in a number of features from the description encountered in the paediatric literature and seem to warrant special discussion.

\section{Case Reports}

Case 1. St. McH., the first of twins (birth weight $3 \mathrm{lb} .12 \mathrm{oz}$.), was admitted to the unit for premature infants two and three-quarters hours after delivery. On examination nothing abnormal was noted. The baby progressed satisfactorily. On the seventeenth day a small raised area of reddish induration was seen at the lower edge of the umbilicus and a few smaller papules of 3 to $4 \mathrm{~mm}$. diameter were found in the suprapubic area. The rash was regarded as a coccal infection and 250,000 units of penicillin were given intramuscularly. During the following five days the number of reddish brown papules grew: the ones already present had increased up to a diameter of $15 \mathrm{~mm}$. Desquamation around the edge and on the surface of the papules had started and a number of them had merged forming a raised red area of map-like configuration surrounding the umbilicus: further nodules had appeared on the penis, thigh and both groins. An area of dusky red induration desquamating at the edge had involved the whole circumference of 
the prepuce (Fig. 1). The scrotum and perianal region were free from the eruption. The clinical diagnosis of skin thrush was made and confirmed by examination of skin scrapings and culture. No thrush of the oral mucosa could be found and repeated cultures of the faeces did not reveal monilia. Local treatment with $1^{\circ}$. a aqueous solution of gentian violet was carried out and cleared the infection within three days. An interesting feature was that the areas of healed thrush lesion did not stain with gentian violet, but the surrounding normal skin showed the typical bluish violet discoloration.

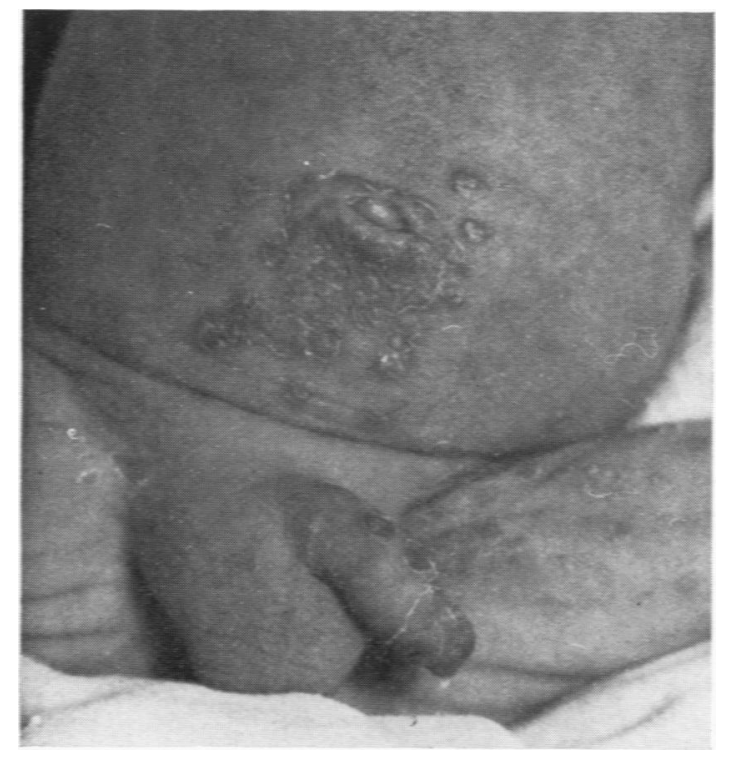

FIG. 1.-Skin thrush of lower abdominal wall. groins, inner aspect of thighs and penis. The lesions show the characteristic peripheral desquamation.

Case 2. L. McH., twin sister of Case 1 (birth weight $4 \mathrm{lb} .7 \mathrm{oz}$.) was admitted at the same time as her brother. On clinical examination she appeared a normal but small infant with some signs of prematurity. On the eighteenth day she developed a number of small red papules on the skin of the lower part of the abdomen, and, as these were thought to be septic, 250,000 units of penicillin were administered intramuscularly. During the ensuing few days the rash spread to the groin and vulva. The correct diagnosis was then made, and confirmed by laboratory methods. No thrush of the mouth could be detected nor was there any infection of the buttocks noticed. Neither vaginal secretion nor faeces contained monilia. The eruption cleared after treatment with $1^{\circ}$ a aqueous solution of gentian violet. Nine days later there was a recurrence of the skin manifestations but the response to reapplication of gentian violet was rapid. The baby's progress had been good throughout.

Case 3. P.T. (birth weight 2 lb.) was admitted to the unit two hours after delivery, and in spite of her low weight was relatively active. There was marked oedema. The infant was nursed according to established principles and seemed to develop satisfactorily. At the age of 9 weeks, and at the same time as the monilia infection occurred in the twins, she showed an eruption of small red nodules on the skin of the abdomen and thighs. Skin scrapings contained Monilia albicans but faeces remained persistently negative. There was no thrush of the mouth present. Treatment with gentian violet was successful. No penicillin had been given in this case.

Case 4. P.A. (birth weight $5 \mathrm{lb} .13 \mathrm{oz}$.) was admitted to the unit at the age of 14 days because of excessive initial weight loss, jaundice and anaemia. On admission it was found that the baby also had extensive paronychiae of both hands. A heavy growth of Staphylococcus aureus, only slightly sensitive to penicillin, was obtained from the infected fingers. Treatment with large doses of penicillin was given for a few days but improvement was only temporary and a fortnight after admission the paronychiae showed some exacerbation with an accumulation of pus necessitating incision. A three days' course of penicillin was again prescribed. The infection subsided but five days later skin thrush was noticed in the suprapubic area and then spread to the penis and prepuce. The monilia infection appeared on the same day as in Case 3.

As the moniliasis of the skin appeared in four babies in the course of one week efforts were made to trace the source of infection, particularly as only the twins shared one large cubicle, the other two infants being in separate ones at opposite ends of the unit and attended by different nurses during the day, but not at night. Utensils, oil, napkins and garments worn close to the skin were searched unsuccessfully for the presence of monilia, and though the origin of the infection remained undetected no further cases occurred.

Case 5. J.B., one of male twins (birth weight $3 \mathrm{lb}$. $8 \mathrm{oz}$.), delivered in a large obstetric department, developed severe staphylococcal conjunctivitis at the age of 14 days. He was given penicillin systemically for several days; at the end of this period he developed a rash on both thighs and lower abdomen, the anal region and buttocks remaining free. There was no moniliasis of the mouth. Skin scrapings confirmed the clinical diagnosis.

Six further cases were observed in the course of a year among infants attending a welfare centre. They were all under 8 weeks old, four of them showed the monilial infection on the skin of the lower abdominal wall, the groins and thighs. Two had lesions on the buttocks and these had also thrush of the mouth and positive cultures from the faeces. None of them had received an antibiotic preceding the appearance of the skin infection. One case of this group who presented an unusual reaction is described in detail.

Case 6. S.W. (birth weight $7 \mathrm{lb} .10 \mathrm{oz}$.) had a normal delivery. At 3 weeks she had mild intertrigo of the left 


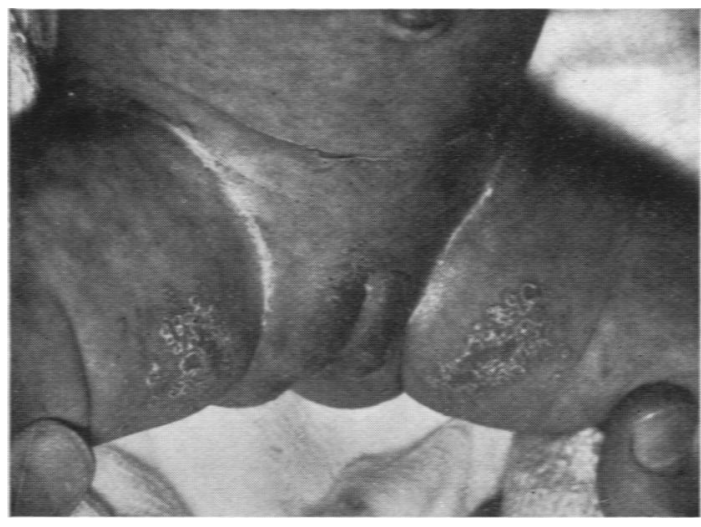

Fig. 2.-Thrush on the skin of thighs and labia, as well as the exanthemitous moniliid on the remainder of the visible skin.

axilla but otherwise was doing well. When 5 weeks old she was brought to the clinic because of a skin eruption on the inner aspect of both thighs and vulva which had persisted for at least one week, and an exanthematous dusky red rash very similar to a drug rash, involving the whole of the trunk and the limbs, which had developed on the day preceding her visit. The lesions on the thighs were of a papular nature, confluent with desquamating edges: the skin of the labia was brownish red, indurated, and slightly raised above the level of the normal skin. The area of induration showed some flaking on the outer edge and terminated abruptly in a distinct line at the inner surface (Fig. 2). Skin scrapings contained large numbers of moniliae but culture from the faeces was negative. After three days' treatment with $1 \%$ aqueous solution of gentian violet the infection had completely subsided and the erythematous rash faded. The normal skin surrounding the thrush lesion was stained blue and revealed the exact outline of the healed infection which had not taken up the dye.

In all cases healing seemed to take place by a process of progressive desquamation until a normal skin surface had re-established itself.

A convenient way of demonstrating the presence of monilia is to obtain scrapings from the edge of the lesion, where desquamating epithelium yields even to the light touch of the scalpel blade, and to deposit the squames obtained on a glass slide, a second glass slide then being pressed firmly on the first one. By moving the two slides in opposite directions, while still under pressure, the larger clumps of cells are easily broken up and monilia spores and pseudo-mycelia can then be found in a Gram stain without difficulty (Fig. 3). This preliminary investigation is then complemented by cultural methods.

\section{Discussion}

Ibrahim (1911), probably the first to describe thrush of the skin in young infants, reported six cases, all of which occurred in babies under 1 month of age. The lesions found by him were intact or ruptured vesicles, localized in the perianal region, buttocks, thighs and lower abdomen. Extensive oral thrush was noticed in every infant, and culture media inoculated with faecal material gave a lush growth of the yeast. Infection of the skin through monilia excreted in the faeces was therefore regarded as the only mode of spread and this idea has since then been generally accepted.

In this series, however, nine of the 11 cases had no thrush in the oral cavity, there were no moniliae in the faeces and the eruption was limited to the lower abdomen, inguinal folds and genitalia with omission of the perianal region and buttocks. The appearance of skin thrush in four infants within a short space of time in one unit, but nursed in completely separate rooms, suggests a different mode of transmission.

Ludlam and Henderson (1942) in their study of neonatal thrush in the mouth found an incidence of $5.2 \%$ in full term and $18.5 \%$ in premature infants. All of a small number of these babies whose stools were examined had faecal moniliasis. But in spite of the high rate of infection among the newborn infants no cases of skin thrush were reported. These authors found Monilia albicans in $33 \%$ of the nurses

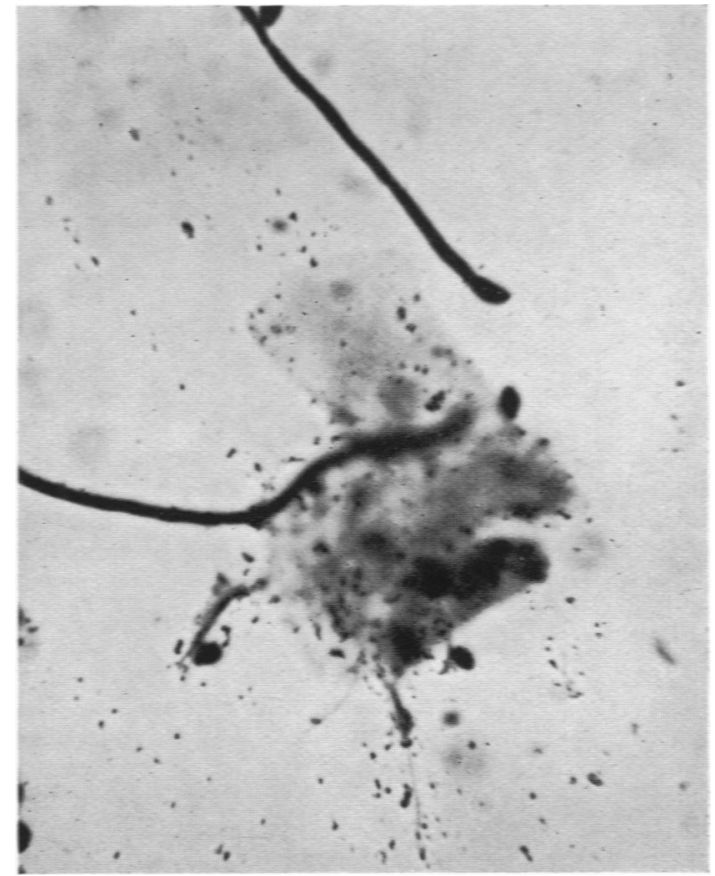

FIG. 3.-Monilia demonstrated in skin scrapings (Gram stained) by the method described. 
and in a relatively small number on their fingers. There is, therefore, the strong possibility that the skin, at least in some of the cases, is infected by a nurse's or mother's hands. Intertrigo, maceration of the very fine and sensitive skin of young infants (our cases were all under 2 months of age) as well as friction due to a rough napkin are probably contributing factors.

During the small epidemic among the premature infants the rapid though localized spread as well as the tendency towards recurrence may well have been due to the large doses of penicillin they received at the onset of the infection. The appearance and extension of the monilia manifestations in Case 5 followed parenteral penicillin administration.

The inability of the affected skin to retain the gentian violet dye, which was so striking in practically all cases, is probably due to the prolonged desquamative process by which healing occurs and by which the stained epithelia are constantly shed.

The occurrence of trichophytides as well as moniliids in adults is not unusual and the eruptions, which may vary from widespread papular or vesicular manifestations to scarlatiniform, morbilliform or erythema multiforme-like rashes, are viewed as an expression of sensitization to toxic products originating from the primary fungus infection. The cyanotic red exanthem observed in Case 6 has to be regarded as moniliid and has not, to our knowledge, been previously reported in skin moniliasis of infancy.

\section{Summary}

Eleven cases of skin thrush in infants under 2 months of age are reported, four of which occurred as a small epidemic outbreak in a premature unit.

There was no oral and faecal moniliasis except in two of the babies.

The possible sources of infection and mode of spread are discussed.

A moniliid eruption in a 5-week-old infant is described.

My thanks are due to Professor Wilfrid Gaisford for his interest and most helpful criticism, to Dr. R. F. Jennison and his staff at St. Mary`s Hospital and Mr. A. Payne at the Duchess of York Hospital for their help with the laboratory investigations and to Mr. G. Ward for the photographs.

\section{REFERENCES}

Ibrahim, J. (1911). Arch. Kinderheilk., 55. 91.

Lederer, $\mathbf{H}$. and McLaren Todd, R. (1949). Archives of Disease in Childhood, 24, 200.

Ludlam, G. B. and Henderson, J. L. (1942). Lancet, 1, 64.

Mayer, J. B., Götz, H. and Seitz, G. (1951). Ann. paediat., Basel, $177,213$.

Nikolowski, W. (1953). Dtsch. med. Hschr., 78, 553. 